\title{
What Is Construction Grammar?
}

Many thanks for inviting me to this wonderful event. It is a great honor for me to be here and speak to you. I wish to express my sincere gratitude to Professor Thomas Li and to all volunteers who have been involved in the preparations for this meeting. I have never been to the China International Forum on Cognitive Linguistics (CIFCL) before, but I am certainly not a stranger to it. Since many of the recorded lectures are available on the internet, I have been able to download and listen to wonderful colleagues like Ewa Dąbrowska, Stefan Th. Gries, or Mark Turner. It is an immense privilege to be invited to follow in their footsteps, and I want you to know that I truly appreciate the honor.

What is this lecture series all about? First of all, the lectures will address the general issue of language change, but they will do so by adopting a perspective that differs from other approaches to historical linguistics. Specifically, I will draw on the theoretical framework of Construction Grammar, in order to explore how a constructional view can help us understand certain aspects of language change that other frameworks find difficult to explain. How can a constructional view help us make progress in the study of how languages develop over time?

In this context, it will be necessary to spell out how a constructional view differs in its assumptions from other theoretical approaches to language change. In Cognitive Linguistics, we have a long tradition of comparing our views to generative and formalist views, and often this goes along with a narrative of conflict between theories. That is not exactly what I will be after in these lectures. I think there are differences worth exploring between approaches that lie within the cognitive-functional enterprise. For example, how does Diachronic Construction Grammar differ in its assumptions from an approach such as grammaticalization theory? It is a worthwhile enterprise to spell out the assumptions and to discuss aspects that are problematic and at this stage unresolved.

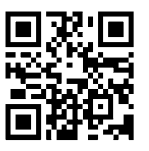

All original audio-recordings and other supplementary material, such as any hand-outs and powerpoint presentations for the lecture series, have been made available online and are referenced via unique Dor numbers on the website www.figshare.com. They may be accessed via this QR code and the following dynamic link: https://doi.org/10.6o84/mg.figshare.1369o93o. 
In my talks, I also want to discuss methodological aspects. What methods can be used for the constructional study of language change? I will be discussing a range of different techniques, mostly corpus-based techniques, but also some experimental methods, that can be exploited within the project of Diachronic Construction Grammar. Of course, a large part of that will be a discussion of the results that we can obtain on the basis of these methods. What do we learn, at the end of the day, when we apply these methods?

My main goal for this lecture series is to give you a general overview of what Diachronic Construction Grammar tries to accomplish. That overview is offered from my personal perspective, which is necessarily subjective. Depending on who you ask, you might hear very different points of view on certain aspects of what I will discuss. As always in science, I would encourage you to adopt whatever you find useful from my discussion and engage critically with whatever you find unconvincing or worthy of criticism. In the ten lectures I will draw on ideas about Diachronic Construction Grammar and language more generally, that I have tried to express in three different books that I have written.

My book Germanic Future Constructions (Hilpert 2008) reflects the way I think about constructions and their associations with lexical items, as well as how these patterns of associations may shift over time. In the lectures, that idea will inform the discussion in several places. In Constructional Change in English (Hilpert 2013), I tried to develop a general account of how language change can be understood from a constructional point of view. I also discussed a variety of different corpus-based methods for the analysis of constructional change.

The third book, which has just come out in a second edition, is Construction Grammar and Its Application to English (Hilpert 2014/2019). That book is my attempt to summarize in general terms what Construction Grammar is all about and what sets it apart from other linguistic theories. While the ten lectures will take these works as a backdrop, I also need to point out that my thinking on these issues has not developed in a vacuum. There are a number of colleagues who I have been lucky enough to collaborate with and whose ideas will therefore inform the discussion. I will make reference to research that I have been doing together with Stefan Th. Gries, with Florent Perek, with David Correia Saavedra, and with Susanne Flach. My views on Diachronic Construction Grammar have also been strongly influenced by other people that you know, Adele Goldberg, Elizabeth Traugott, Graeme Trousdale, and Holger Diessel. In these lectures, I will sometimes point out issues where I think we disagree, but in principle our views are very similar, so there is a lot more that we have in common than what we see differently. But even so, I think it is always useful to talk about the issues where you actually disagree. 


\section{Ten lectures on Diachronic Construction Grammar}

1. What is Construction Grammar?

2. Taking a constructional approach to language change

3. Three open questions in Diachronic Construction Grammar

4. Shifts in collocational preferences

5. How constructional networks grow and fade

6. Competition in constructional change

7. Differentiation and attraction in constructional change

8. The asymmetric priming hypothesis

9. The upward strengthening hypothesis

10. Constructional change and distributional semantics

FIGURE 1

Coming now to the structure of the lecture series, one thing I would like to say is that I wanted these lectures to be accessible to all of you, regardless of your background. I assume some familiarity with basic notions in Cognitive Linguistics, but not much more. This means that some of you will undoubtedly recognize ideas that are quite familiar to you, but I promise to contextualize them in such a way that hopefully, I will make you see them in a new way. We'll start today with two lectures that will provide the theoretical basis for everything else that I have to say.

In this first lecture, which simply has the title What is Construction Grammar?, I will try to clarify my assumptions and define my theoretical terms. The lecture for this afternoon is entitled Taking a construction approach to language change. In that lecture, we will move into the subject of language change. Once these ideas are in place, the third lecture, on Three open questions in Diachronic Construction Grammar, will take us to a different area of debate, where current research has not yet found a consensus. The first three lectures here can be seen as the theoretical groundwork for the rest of the lectures.

Lectures 4 to 7 will exemplify different ways of dealing with the open questions and with the debates in Diachronic Construction Grammar. Lecture 4 will focus on the relation between constructions and lexical items, and more specifically, shifts in the collocational preferences of constructions, and what we can learn from those shifts.

Lecture 5 takes up the idea of a constructional network, and it will discuss how constructional networks may develop over time. How do these networks grow? How do they branch out and how do they fade away in the end? 
Lecture 6 addresses the notion of competition in constructional change. Some constructions are mutual alternatives, so that they can be seen as competing with one another. Much research in sociolinguistics actually draws on the metaphor of competition between linguistic forms, so I will explore what this idea implies for constructional theories of constructional change.

Lecture 7 will look at two different trajectories of constructional change, namely differentiation, constructions becoming more different, and attraction, constructions becoming more similar to one another. I will look at the reasons for differentiation and attraction and how developments of this kind can be studied.

Lecture 8 and 9 will bring us back into the realm of linguistic theory. The big question that they both try to address is why languages change in the way they do. Lecture 8 addresses what I call the asymmetric priming hypothesis, which tries to explain the unidirectionality of semantic change in grammaticalization. Lecture 9, on the upward strengthening hypothesis, addresses the emergence and entrenchment of grammatical markers from a constructional perspective.

In the final lecture, I will discuss recent connections between work in Diachronic Construction Grammar and corpus-linguistic work on what is called distributional semantics. I will close with a discussion of how theory and methodology can come together in useful ways.

With this broad overview in place, let me come to the first lecture - What is Construction Grammar? A very short answer to that question is that Construction Grammar is a theory of what speakers know when they know

\section{What speakers have to know}

- must know words

- dog, submarine, probably, you, should, etc.

- what they mean, how they sound

- must know that there are different kinds of words

- red is an adjective, tasty is an adjective as well, lobster is a noun, etc.

- must know how to put words together

- red can be combined with ball

- many cannot be combined with milk

- John saw Mary is ok, Saw John Mary is not, but It's John Mary saw is

- must be able to put the right endings on words

- John walk-s, two dog-s

- must be able to understand newly coined words

- festive-ness, under-whelm

- must know that sometimes more is meant than is said

- General Motors were able to increase production in the second quarter.

- I don't know if that is a good idea.

- must know idiomatic expressions

- I'm all ears, let's take a break, we really hit it off, ... 
a language, that is, when they know how to produce and process language. In other words, Construction Grammar has the same goals as any other cognitive theory of language. What is it that speakers have to know? There actually is not much disagreement. Anyone studying language would have to come to terms with certain things that speakers simply have to know in order to engage in a conversation.

Here's a list of things that speakers of any language have to know in order to talk. I have given you examples from English here, but the main ideas would be the same for really any language that you study. Speakers have to know words, what they mean and how they sound. They must know that there are different kinds of words. For English, there are different word classes like adjectives, nouns and verbs, and speakers know that these word classes behave differently. Speakers must know how to put words together, how to form phrases and sentences out of the lexical words that they know. Speakers must be able to put the right endings on words. If your language is morphological, if it has lots of inflections, then you have to know how to put words together from these smaller materials. Speakers must be able to understand newly coined words. There are derivational morphological word formation processes that enable speakers to produce new words, and hearers have to be able to understand what these new words mean. Speakers must know that sometimes more is meant than what is said. For example, if I tell you I do not know if that is a good idea, I do not express just my ignorance on a certain point, but rather I tell you that this is not a good idea. That meaning is not right there in the words, but that is something that you infer. Lastly, speakers must know idiomatic expressions, combinations of words that have non-compositional meanings that convey a kind of meaning that cannot be inferred by understanding the parts of the expression.

In this laundry list of things that speakers have to know, you recognize some traditional domains of linguistic research. How do speakers know what words can be put together? That is the domain of syntax. How speakers are able to produce new words? That falls into the domain of linguistic morphology. Understanding that more is meant than what is said, that is what we usually study in pragmatics. Many linguistic theories view these points as distinct, as falling into different areas of linguistic knowledge, different modules even of linguistic knowledge. Construction Grammar is different in this regard because Construction Grammar posits that all types of linguistic knowledge can be seen as being of the same type, as being cut from the same cloth.

What speakers have to know, from the perspective of Construction Grammar, can be expressed much more concisely than this list, namely that speakers must know constructions. In other words, all the items from our 
long list of things that speakers have to know, lexical items, word classes, syntactic patterns, and so on and so forth, can and should be re-conceptualized as knowledge of constructions. Constructions are defined as form-meaning pairings - symbolic units that pair linguistic form with conceptual meaning. This is the underlying basic proposal that Construction Grammar makes. To properly appreciate how this proposal works, what it implies and where it maybe has its limits, I want to flesh it out and discuss ten basic ideas that I view as fundamental for Construction Grammar. If you've understood those ideas, then you're really in a good position to assess what the constructional enterprise is all about.

\section{Basic idea \#1}

- All of linguistic knowledge is a network of form-meaning pairs constructions and nothing else in addition.

The totality of our knowledge of language is captured by a network of constructions: a 'construct-i-con.'

Goldberg (2003: 219)
A speaker's knowledge of grammatical patterns resides in a vast inventory of symbolic assemblies ranging widely along the parameters of schematicity and symbolic complexity.

Langacker (2013: 24)

FIGURE 3

Let's jump right in with basic idea \#1. All of linguistic knowledge is a network of form-meaning pairs - constructions and nothing else in addition. This idea re-captures what I have been saying so far, especially the last bit there, "nothing else in addition', that is sometimes contested. There are colleagues who may be generally sympathetic towards Construction Grammar, but who would feel skeptical about it. Can you really capture everything about linguistic knowledge with form-meaning pairs? Don't you need some kind of abstract syntax? Don't you need some overarching pragmatic principles? What about phonemes? The list goes on, but I would like to stress that this idea is to be taken very literally. Our goal as Construction Grammarians is to explain everything in language through constructions and nothing else.

To give some emphasis to this, I have given you two quotes here, one from Adele Goldberg and the other from Ronald Langacker, both state exactly this idea. Adele Goldberg (2003: 219) states that 'the totality of our knowledge of language is captured by a network of constructions: a 'construct-i-con'. Langacker 
(2013: 24) formulates it as follows: 'A speaker's knowledge of grammatical patterns resides in a vast inventory of symbolic assemblies ranging widely across the parameters of schematicity and symbolic complexity.' This idea forms the very basis of everything else I am going to say.

\section{Basic idea \#2}

- The basic unit of linguistic knowledge are symbolic pairings of form and meaning.

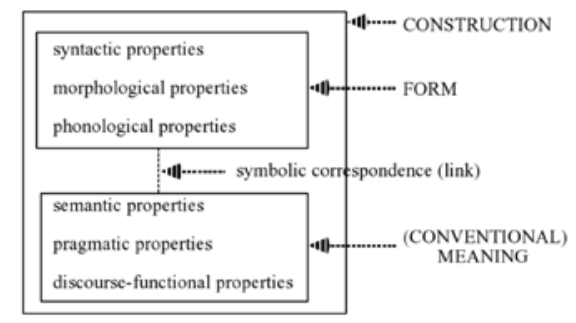

FIGURE 4

The basic unit of linguistic knowledge are symbolic pairings of form and meaning. This idea captures how we define what a construction is. Constructions, the basic units of linguistic knowledge, are defined as pairings of form and meaning. Form, as you see in the diagram on the slide, taken from the work of Croft and Cruse (2004: 258), comprises phonological structure, morphological structures and syntactic structure. Meaning includes semantic, pragmatic and discourse-functional meaning. So there are different shades of form and meaning, and these are linked and connected through the symbolic link, which is an association that is typically arbitrary and established through convention. The diagram on this slide represents a broad consensus in the field, although I will have more to say about it as we go along.

For now, let's carry on, here is basic idea \# 3 . That is the notion that 'constructions vary in terms of their degrees of complexity and schematicity.' You've seen this already in the quote by Ronald Langacker that I gave you earlier, here we just flesh it out a little more.

This is a diagram that I have adapted from Langacker's work. Langacker (2005: 108) conveys the idea that constructions vary along two axes, the axis of complexity, that is the $x$-axis here, and the axis of schematicity, that is the $y$-axis. The $x$-axis represents a continuum from mono-morphemic simple constructions to more complex patterns that have several different parts. The $y$-axis represents another continuum that ranges from very specific constructions to 


\section{Basic idea \#3}

- Constructions vary in terms of their degrees of complexity and schematicity.

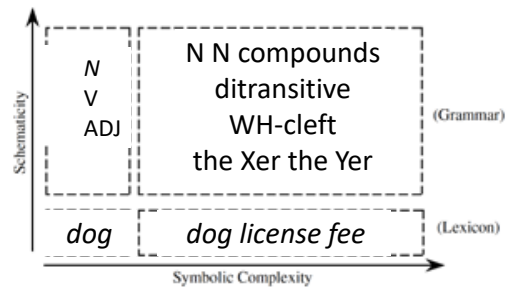

more schematic and abstract constructions. This means that on the $y$-axis we have a continuum between lexis at the bottom and grammar at the top, lexis being more specific in meaning, and grammar more schematic.

Let me illustrate this diagram a little further with concrete examples. A construction that is low in complexity and low in schematicity would be a mono-morphemic word such as dog. Constructions that are low in complexity but high in schematicity are schematic word classes such as nouns, verbs, or adjectives, which according to basic ideas \#1 and \#2 we would think of as constructions. They're simple but very general, abstract and schematic constructions. If we move on to constructions that are low in schematicity but high in complexity, we get compound words such as for example, dog license fee, which are internally complex. They have structure. But as far as their meaning goes, they are highly specific. That leads to the fourth possible combination, constructions that are both complex and schematic. Here we have grammatical constructions in the traditional sense, units like noun-noun compounds, the ditransitive construction, cleft sentences, constructions like the comparative correlative construction, the Xer the Yer, and so on and so forth. One idea that I will come back to several times in this lecture series is how we can tell where exactly in this coordinate system of complexity and schematicity we should locate a specific construction that we are talking about and that we are studying.

Constructions are idiosyncratic, that is, they are to some extent unpredictable. What this means is that as a learner of a language, you cannot deduce how they work from first principles, but rather you have to learn and memorize them. They have characteristics that you cannot figure out or deduce logically, even when you have a lot of knowledge about the language already. 


\section{Basic idea \#4}

- Constructions are idiosyncratic.

- Constructional meanings are often non-compositional.

- What's this fly doing in my soup?

- Constructional forms are often not predictable from general rules.

- The more you think about it, the less sense it makes.

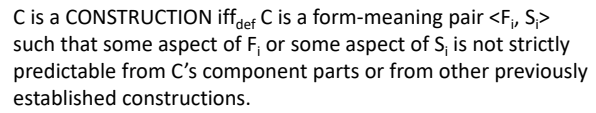

Goldberg (1995: 4)

FIGURE 6

Constructional idiosyncrasies have figured prominently in the work of Chuck Fillmore, which is foundational for Construction Grammar. Idiosyncrasies may pertain to both linguistic meaning and linguistic form. With regard to meaning, they give rise to the phenomenon of non-compositionality. For example, in an utterance like 'What's this fly doing in my soup?', we understand that the speaker is not just asking a question but actually making a complaint. This meaning component is not expressed by the individual words, but rather it emerges from the holistic properties of the construction. Idiosyncrasies with regard to form mean that we have constructions whose form is not predictable from general morphosyntactic rules. This is the case, for example, in the comparative correlative construction: The more you think about it, the less sense it makes. Syntactically, this sentence does not look like any other construction of English that we might have come across. This is something that we need to learn and memorize as second language learners of English.

The basic idea of constructional idiosyncrasies is so central for Construction Grammar that it has made its way into one of the most influential definitions of what constructions are, namely the definition proposed by Adele Goldberg in her book on argument structure constructions (1995:4):

$\mathrm{C}$ is a construction if and only if that construction is a form-meaning pair, such that some aspect of the form or some aspect of the meaning is not strictly predictable from the constructions' component parts or from other previously established constructions. 


\section{Basic idea \#5}

- All constructions, including schematic syntactic patterns, carry meaning.

- She sliced the box open.

[A]rgument-structure constructions provide the direct link between surface form and general aspects of the interpretation, such as [...] someone causing something to change state.

Goldberg (2003: 221)

FIGURE 7

One question that you might ask here is why construction grammarians make such a big deal out of idiosyncrasies. If you look at authentic natural language use, you'll actually find that idiosyncrasies are ubiquitous. You find them everywhere. You run into them a lot more than traditional accounts of language structure would have us believe, and that is why it is such an important idea.

I am coming to basic idea \# 5 , which is the idea that all constructions, including schematic syntactic patterns, carry meaning. Many working linguists will tell you that during their years of study, there have been two or three studies that have opened their eyes towards a certain aspect of language and that made a lasting impact on the way they came to think about language. For me, one such text was Adele Goldberg's work on argument structure constructions. That work makes the general point that syntactic patterns like the ditransitive construction or the resultative construction are not just formal syntactic patterns. Instead, they are symbolic units that carry meaning. In a sentence like She sliced the box open, the structure of that sentence conveys the meaning that as the result of her slicing, the box opened. Today, it seems very obvious to me that the syntactic patterns can convey these ideas. But before reading Goldberg, I thought that syntax is about arranging words into phrases and that meaning chiefly resides in the words, not in the patterns. In the words of Goldberg herself, argument structure constructions provide the direct link between surface form and general aspects of the interpretation, such as someone causing something to change state (2003: 221). So for me, this idea substantially changed how I viewed language.

Basic idea \#6 is the so-called 'principle of coercion'. When there is a conflict between lexical meaning and the meaning of grammatical constructions, 


\section{Basic idea \#6}

- When there is conflict between lexical meaning and the meaning of grammatical constructions, the construction produces a coercion effect.

- Two beers, please.

If a lexical item is semantically incompatible with its morphosyntactic context, the meaning of the lexical item conforms to the meaning of the structure in which it is embedded.

Michaelis (2004: 25)

\section{Basic idea \#7}

- Grammatical categories are the outcome of speakers generalizing over instances of language use.

- SUBJECT is an abstraction over the agentive roles that occur in the transitive construction, the ditransitive construction, and in other clausal constructions. Speakers do not necessarily perceive these as the same.

No schematic syntactic category is ever an independent unit of grammatical representation.

Croft (2001: 55)

FIGURE 9

the construction produces a coercion effect. Let me illustrate this. In English there are nouns such as beer, which function as so-called mass nouns. They denote substances that are not easily counted. When I put them in a context where they are treated as countable, I do something to their meaning. Take an utterance such as Two beers, please. Instead of treating beer like the mass noun that it is, I convey that I would like two units of beer, that is, two glasses of beer or two bottles of beer. This is the principle of coercion by construction, which was formulated by Laura Michaelis as follows (2004: 25): If a lexical item is semantically incompatible with its morphosyntactic context (for example beer, which as a mass noun is incompatible with the context of the plural, so that an 
uncountable is made countable through the plural), the meaning of the lexical item conforms to the meaning of the structure in which it is embedded. In other words, the construction wins. If there is conflict between a lexical item and its constructional context, then the construction wins out.

Basic idea $\#_{7}$ is a notion that is now generally accepted in usage-based linguistics, but that I found quite far-reaching when I first encountered it in the work by Bill Croft, in his book (2001) on Radical Construction Grammar. What he argued specifically was that grammatical categories are the outcome of speakers generalizing over instances of language use. That is, a grammatical category such as subject, for instance, is not a grammatical primitive or a unit that is basic, but rather it is the opposite. It is an emergent phenomenon. It is a generalization or an abstraction over the agentive roles that occur in the transitive construction (John kicked the ball), the ditransitive construction (John gave Mary the book), or other clausal constructions (John promised to pick me up). Speakers do not necessarily perceive these as exactly the same, but they perceive these roles as similar enough to instantiate a broad category of subject in English. Across languages these categories are not the same. The same holds for other notions that we as linguists are perhaps used to seeing as very basic. We have been trained to work with categories such as nouns, verbs, cases like dative and accusative, subordinate clauses, and so on. All of these high level generalizations are really emergent phenomena. They're not grammatical primitives, but rather they are the outcome of your experience with many tokens of language use.

Croft (2001: 55) formulates this idea like this: No schematic syntactic category is ever an independent unit of grammatical representation. Every category is the outcome of speakers hearing many instances of language use and drawing a generalization from that experience. This is important, since it means that we have to ask ourselves this: When is a phenomenon that we are studying a construction? Can we assume that speakers have drawn a generalization from the input that they have had? What is the evidence for this? We will come back to this question a couple of times.

Basic idea $\# 8$ is an idea that is near and dear to my heart. Let me explain what it is about. The idea is that constructional meaning is reflected in associations between syntactic patterns and lexical elements. The sentence John gave Mary the book instantiates what we call the ditransitive construction, which has as its basic meaning the idea of a transfer. It therefore comes as no surprise that the verb give is the one verb that is most strongly attracted to that construction, and that is most strongly associated with that construction. A similar point can be made for the way-construction, He elbowed his way through the crowd. This construction conveys the idea of movement along a path that is difficult and laborious. In that construction we tend to find verbs such as the denominal 


\section{Basic idea \#8}

- Constructional meaning is reflected in associations between syntactic patterns and lexical elements.

- John gave Mary the book.

- He elbowed his way through the crowd.

If syntactic structures served as meaningless templates waiting for the insertion of lexical material, no significant associations between these templates and specific verbs would be expected.

Stefanowitsch \& Gries (2003: 236)

FIGURE 10

verb elbow. It means that you create your path, pushing other people with your elbows, for example when you enter the subway. This conveys exactly the kind of difficult movement that the construction commonly expresses. If you like, you can see this as a kind of harmony in meaning between the meaning of a construction and lexical items that occur in that construction. You could see this as another piece of evidence that syntax is in fact meaningful. This slide shows a quote by Stefanowitsch and Gries (2003: 236): If syntactic structures served as meaningless templates waiting for the insertion of lexical material, no significant associations between these templates and specific verbs would be expected. If syntax were really just a set of rules of putting words together, then why do we see these harmonious patterns of specific verbs being attracted to specific syntactic contexts?

\section{Basic idea \#9}

- Knowledge of constructions is usage-based. Every single usage event produces a change in the network of constructions.

Central to the usage-based position is the hypothesis that instances of use impact the cognitive representation of language.

Bybee (2010: 14) 
Basic idea \# 9 is that knowledge of constructions is usage-based. Joan Bybee, one of the principal architects of usage-based linguistics, formulates it in this way (2010: 14): Central to the usage-based position is the hypothesis that instances of use impact the cognitive representation of language. This means that every instance of language use leaves a little imprint on our knowledge of language. This may seem strange at first because it seems to imply that we actually remember everything we've ever heard, every conversation that we've ever been in. I do not know about you, but I forget things all the time, including my keys and my boxed lunch. I have three children and sometimes when I talk to them, I have to go through all three names before I finally get to the right one. I like to think that I am still a normal human being. Normal human beings sometimes forget things. Now with language, most language that we encounter instantiates words and patterns that we have heard lots and lots of times. Hearing the same structure and the same words once again will not change our representations a whole lot. By contrast, hearing structures that deviate from what you've heard before will actually force you to adjust your linguistic representations, just a little bit. Perhaps when you're listening to me, you may need to get used to my accent, the way I pronounce my words, and the melody of my phrases. This is day one, so you're still adjusting and you're still trying to figure out my vowels and other aspects of the way I say things. But you'll see that over day two, day three and day four, you will gradually settle into the way of processing my speech. You will find it easier to follow. That means that your cognitive representations of language have indeed changed just a little bit, just by listening to me. That is the core idea of usage-based linguistics, and that brings me to basic idea\#10, which is the bedrock of any usage-based understanding of language, and which I find best expressed in the work of Michael Tomasello.

\section{Basic idea \#10}

- Language draws on domain-general socio-cognitive processes, including categorization, association, routinization, generalization, schematization, joint attention, statistical learning, analogy, metaphor, and others.

[C]hildren acquire all linguistic symbols of whatever type with one set of general cognitive processes.

Tomasello (2005: 193) 
The idea is that language draws on domain-general socio-cognitive processes, including categorization, association, routinization, generalization, schematization, joint attention, statistical learning, analogy, metaphor, and others. The list goes further, but these are the main processes. Whereas, for example, generative linguists assume that we come into this world with language-specific knowledge, a universal grammar, cognitive linguists and construction grammarians work on the assumption that a specific combination of general cognitive and social skills is actually enough of a basis for language learning. This combination of skills explains why humans have language and why other animal species do not. No one disputes that humans and other primates differ in this regard. We have language, but other species do not. We need an explanation for that, and the usage-based explanation is that our configuration of socio-cognitive skills is a different one. The way Tomasello (2005: 193) puts it is that $[C]$ hildren acquire all linguistic symbols of whatever type with one set of general cognitive processes.

These ten ideas are points that I am prepared to defend against any criticism that could be leveled against them. I assume them as a foundation of everything else that I will have to say in this lecture series. Seeing as these points are rather general, I would like us to move on with questions that go into some more detail with regard to constructions, what they are and how we can tell whether a linguistic form is actually a construction.

It is perhaps easy to identify the way-construction or the ditransitive construction, but beyond that, how can constructions be identified? One question that my students ask all the time is how do I know if something is a construction? Is everything a construction? I want to go over four strategies that you can apply when you're asking yourself whether you are dealing with a construction.

\section{strategy \#1}

- Does it have characteristics that deviate from canonical patterns?

- I have waited many a day for this to happen.

- a six year old child

- If he gets here earlier, all the better.

- I kid you not.

- Into the room walked Noam Chomsky.

- I am bitter enemies with John. 
The first strategy relates to basic idea \#4, the idea that constructions are idiosyncratic, that there is something unpredictable about them. When you're looking at a linguistic structure, do you see characteristics that deviate in some way from canonical patterns of the language that you are studying? Can you figure it out from other regularities that you know, or is there something that you would have to learn and memorize that is specific to this pattern? This slide lists a few examples that show formal idiosyncrasies of this type.

For example, in the utterance I have waited many a day for this to happen, the phrase many a day will look odd to many of you, since it shows an unusual sequence of the quantifier many, the singular indefinite article $a$, and a singular noun. Normally, we expect to see many with a noun that is in the plural, as in many days. Here it is many a day, which is clearly different from canonical English syntax. If you find any deviation of this kind, you know that you're looking at a pattern with some irregularity, which fits our definition of a construction.

\section{strategy \#2}

- Is its meaning non-compositional? Does the whole mean more than the combination of the parts?

- How are you doing!

- During the game he broke a finger.

- We have been best friends since high school.

FIGURE 14

The second related strategy would be to look for non-compositional meanings. If we have a particular example of language use, we can ask ourselves whether its meaning is non-compositional. Does the whole somehow mean more than the combination of the parts? Let's just take a quick look at the first example. The utterance 'How are you doing! may look like a question, but it also has the non-compositional meaning of a greeting formula, and that is a matter of convention. That is something that you cannot figure out on the basis of the words alone. It is something that you have to learn on the basis of contextual information. Whenever the meaning of the whole is more than the meaning of the parts, then you know that you're dealing with a construction. 


\section{strategy \#3}

- Does it have constraints that are idiosyncratic?

- Mary is a smarter lawyer than John.

- * Mary is the smarter lawyer than John.

- The dog over there is asleep.

- * Over there is the asleep dog.

- un-conscious, un-aware, un-cool

- * un-green, ? un-awake, ? un-special

- I brought John a glass of water.

- * I brought the table a glass of water.

FIGURE 15

For strategy \# 3 , you need to ask the following question. Does the pattern that I am looking at have constraints that are idiosyncratic? This strategy, I am ready to admit, is a little tricky, specifically for second language learners of English, because it requires you to manipulate the pattern in several ways, and make judgments about what can and cannot be said. As a linguistic methodology, introspective grammaticality judgments have serious problems, but I think that there are contexts in which they show us something.

Let us take, for example, the utterance The dog over there is asleep. Speakers of English use adjectives like asleep in what is called a predicative construction, in which the adjective follows a form of the verb to be. If you try to use asleep in a different syntactic position, as an attributive adjective, as in *the asleep dog or *the asleep baby, speakers of English will actually give you strange looks, because that is not the way you use this kind of adjective. Asleep does not work in that way. You can't put it before a noun. You have to say the baby is asleep rather than *the asleep baby. Restrictions of this kind mean that we are dealing with a construction. There is an idiosyncrasy about asleep and other related adjectives that has to be learned. The other examples on the slide illustrate the same point with other structures, but the general point in all cases is that whenever a manipulation of a grammatical structure yields an expression that sounds odd or unacceptable, that means that you're looking at a construction. 


\section{strategy \#4}

- Does it have collocational preferences?

- He drives me crazy / insane / bananas / up the wall / ...

- I shall

- return to this topic in chapter 4

- discuss quantum theory in chapter 5

- argue for a constructional account in chapter 6

- ? call you after lunch

- Einstein was the first to fully understand relativity.

- ? Einstein was the first to adequately describe relativity.

- Corpus evidence / psycholinguistic evidence needed for this strategy.

FIGURE 16

Strategy \# 4 brings us back to the basic idea that constructions have collocational preferences and that syntactic patterns tend to be associated with specific lexical items. Whenever we have a syntactic pattern that is not just indiscriminate with regard to the words that it occurs with, but that shows particular associations, then we can say that we found a construction. The question to ask is whether a linguistic form has collocational preferences and what these preferences are. Strong collocational preferences are evident in the case of idioms, such as the drive someone crazy construction. There are a handful of elements that can appear in the final predicative slot of the construction. You can drive someone crazy, you can drive them insane, you can drive them bananas, mad, or up the wall, but that is already more or less the whole spectrum. There are many adjectives or other expressions that do not work in the drive crazy construction. You cannot *drive someone happy or *drive someone sane, so there are limitations, and these limitations instantiate constraints that the construction has.

There are other constructions that are a lot more open with regard to their collocates, but that show nonetheless a recognizable profile of collocational preferences. For example, the English auxiliary shall is strongly associated with lexical verbs such as return, as in I shall return to this topic, or discuss, as in I shall discuss this in chapter five. It is much less associated with other lexical verbs. An utterance such as I shall call you after lunch is possible. But it is less idiomatic than I shall argue or I shall discuss. The last example on this slide illustrates the so-called split infinitive construction in English, Einstein was the first to fully understand relativity. To fully understand is a split infinitive that consists of the infinitive marker to, the verb understand and the adverb fully, 
which so to speak splits the two in half. This construction works well with certain adverbs, but not so well with others. To fully understand sounds good to most speakers, but *to adequately describe does not. It is not ungrammatical, but it is clearly worse. In any case, the bottom line would be that the presence of collocational preferences is a hint that a construction is present. You can try to argue for collocational preferences on the basis of grammaticality judgments, as I have done here, but really the more suitable evidence for this would come from corpus studies or from psycholinguistic experiments. I would also like to add that strategy \# 3 and strategy \# 4 boil down to the same idea: They show preferences and restrictions on a continuum from hard constraints to probabilistic biases.

\section{strategy \#3}

- Does it have constraints that are idiosyncratic?

- Mary is a smarter lawyer than John.

- * Mary is the smarter lawyer than John.

- The dog over there is asleep.

- * Over there is the asleep dog.

- un-conscious, un-aware, un-cool

- * un-green, ? un-awake, ? un-special

- I brought John a glass of water.

- * I brought the table a glass of water.

\section{strategy \#4}

- Does it have collocational preferences?

- He drives me crazy / insane / bananas / up the wall / ...

- I shall

- return to this topic in chapter 4

- discuss quantum theory in chapter 5

- argue for a constructional account in chapter 6

- ? call you after lunch

- Einstein was the first to fully understand relativity.

- ? Einstein was the first to adequately describe relativity.

- Corpus evidence / psycholinguistic evidence needed for this strategy. 
Strategy \#3, which prompts us to ask whether a construction exhibits idiosyncracies, shows hard constraints, manipulations that you absolutely cannot do with a construction.

Strategy \#4 relates to probabilistic preferences. This relates to patterns of co-occurrence that are preferred and dispreferred. Some patterns are highly entrenched, others are possible, but do not work quite as well as others.

Putting it all together then, a linguistic form is a construction if it deviates from canonical patterns, if it shows non-compositional meanings, if it has idiosyncratic constraints, and if it has collocational preferences. I know that there are members of this audience for whom everything I have said so far is well known and perhaps even self-evident, and I thank you for bearing with me up to this point. I would now like to leave the well-trodden paths and discuss ideas that are somewhat more controversial.

Specifically, I would like to talk about five controversies, which represent issues that construction grammarians do not agree on with each other. These controversies, as I see them, are current construction sites of the field, where the architects do not really agree if they want to build a bridge, or maybe rather a tunnel, or perhaps both.

\section{Controversy \#1}

- Complete inheritance vs. redundant representations

- Do speakers cognitively represent grammatical information just once or several times?

- English plural: NOUN-s, cat-s

- Complete inheritance: information is stored only once, at the most abstract level, within general constructions, specific constructions 'inherit' that information

- speakers don't need to represent cats

- Redundant representations: information is stored at several levels of abstraction

- speakers redundantly store frequent plurals such as cats

FIGURE 19

The first controversy is concerned with relations between constructions in the constructional network, that is, the idea that is commonly known as inheritance. Everyone agrees that constructions are connected, but what exactly are the consequences are of these connections? That is a matter of debate, and the principal conflict here is between two views, which we can label "the complete inheritance view" and "the view of redundant representations", respectively. 
The underlying question is whether speakers cognitively represent grammatical information just once, or rather several times.

Let me give you an example. You and I can understand the word cats, cat in the plural form. Is that because we have memorized the word cats, or is it because we know the plural construction that tells us to form cats by adding an -s to the singular form cat? Is it one or the other, or is it perhaps even both? The view from complete inheritance is that anything that you can describe with a generalization does not have to be separately stored and memorized. Complete inheritance means that information is stored only once, namely at the most abstract level within a general construction. Then specific constructions can inherit that information. They can look it up at a higher level of abstraction in the constructional network. By virtue of that, speakers actually do not need to represent cats. You can see that is a very economical and very elegant way of storing information. You can encode lots of information with relatively little storage, but at the same time it raises questions.

The opposing view, the view of redundant representations, is held by researchers like Ewa Dąbrowska (2017), who has actually argued forcefully for it in this very room at the forum. She argues that information is stored at several levels of abstraction. Even though we do not technically need to memorize the word form cats, because it is such a frequent word, we actually cannot avoid remembering it, and we end up with a redundant representation. On that account, information is stored at several levels of abstraction, so speakers redundantly store frequent plurals with their lexical items in addition to a general plural construction.

If you ask me where I stand on the issue, I appreciate Charles Fillmore and his work with all my heart. But here I would side with Ewa Dąbrowska's view, which embodies the perspective of current usage-based linguistics.

\section{Controversy \#2}

- Lower-level constructions vs. higher-order schemas

- Do speakers cognitively represent generalizations across constructions?

- John gave Mary the book - John gave the book to Mary

The surface generalizations hypothesis:

There are typically broader syntactic and semantic generalizations associated with a surface argument structure form than exist between the same surface form and a distinct form that it is hypothesized to be syntactically or semantically derived from.
Since alternation-based generalizations were relied on much more often in the sorting task than constructional ones, it is reasonable to hypothesize that they correspond to stored generalizations. 
Controversy \# 2 brings us back to the continuum of complexity and schematicity that I talked about earlier. How abstract are the generalizations that speakers draw? If you remember the coordinate system that Langacker proposes to us, where exactly do we place a given construction? Is more information represented at low levels of generalization, or are there highly abstract schemas, perhaps even schemas of schemas, or meta-generalizations? Do speakers cognitively represent generalizations across constructions? That is under debate. For example, is there a generalization across the ditransitive construction, John gave Mary the book, and the prepositional dative construction, John gave the book to Mary? You could argue that both express a transfer, so they have things in common, and as human beings, we categorize items that have features in common. Why not? There could be a generalization of this kind. Adele Goldberg has been an advocate of the idea that low-level generalizations are very important. She has formulated the so-called "surface generalizations hypothesis" (Goldberg 2002), which goes as follows. The surface generalizations hypothesis states that

there are typically broader syntactic and semantic generalizations associated with a surface argument structure form than exist between the same surface form and a distinct form that it is hypothesized to be syntactically or semantically derived from.

GOLDBERG 2OO2: 329

Even though the ditransitive and the prepositional dative have features in common, what they have in common is less substantial than what each individual construction has in terms of its individual characteristics. But this does not necessarily mean that speakers do not draw any abstract generalizations. Florent Perek has done empirical work on this, finding that speakers actually use higher-order schemas when they reason about language. When you give them a categorization task, they will draw on generalizations that reach across several constructions. Perek conducted a sorting task and concluded the following (2012: 629): Since alternation-based generalizations were relied on much more often in the sorting task than constructional ones, it is reasonable to hypothesize that they correspond to stored generalizations. This indicates that speakers store alternations of constructions as meta-generalizations. Adele Goldberg and Florent Perek, as some of you might know, are by now co-authors of a series of studies, so their views are not entirely incompatible. I will come back to this issue later in this lecture. 


\section{Controversy \#3}

- Sound and meaning vs. sound, form and meaning In Cognitive Grammar, the form in a form-meaning pairing is
specifically phonological structure. [I]t does not include what
might be called grammatical form.

Langacker (2003: 104)

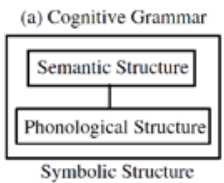

(b) (Radical) Construction Grammar

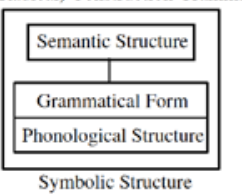

FIGURE 21

Controversy \#3 plays out between Ronald Langacker on the one hand and William Croft on the other, two founders of cognitive linguistics. The issue is how we understand constructions as pairings of form and meaning. Specifically the question is, is form just sound, or is form sound and morphosyntax? Langacker is crystal clear on this (2003: 104): In Cognitive Grammar, the form in a form-meaning pairing is specifically phonological structure. [I]t does not include what might be called grammatical form. The diagram on this slide shows that Croft's Radical Construction Grammar subsumes grammatical, morphosyntactic form under the form side of constructions. Langacker himself only assumes a link between semantic structure and phonological structure. Cognitive Grammar attempts to reduce all linguistic structure to concepts and sounds. It is an ambitious reductionist enterprise. For William Croft, grammatical form is the substance that linguists are working with: words, suffixes and syntactic patterns. It is hard to part with that working material. Can morphosyntax really be reduced to sound? What do we do with part-of-speech constructions like nouns or notions like subject? Can we find sounds that correspond to these categories? Where does Langacker's linking of concepts and sound leave notions such as linear sequence? I will simply leave you with these questions and move on to controversy \#4, the question whether morphemes are constructions or whether they are parts of constructions.

Adele Goldberg has produced several overviews of different construction types, including morphemes like English affixes pre- or -ing. The table on this slide shows different examples of constructions varying in size and complexity, and morphemes are part of that. This view is not generally shared. 


\section{Controversy \#4}

- Morphemes as constructions vs. morphemes as part of constructions

Goldberg (2006: 5)

Booij (2013: 256)

TAsLe 1.1. Examples of constructions, varying in size and complexity

\begin{tabular}{|c|c|}
\hline Morpheme & eg pre, -ing \\
\hline Word & e.g avocado, anaconda, and \\
\hline Complex word & c. \& daredevil, shoo-in \\
\hline Complex word (partially filled) & e.g. [N-s] (for regular plurals) \\
\hline Idiom (filled) & e. going great guns, give the Devil his due \\
\hline Idiom (partially filled) & $\begin{array}{l}\text { e. jog <someone's> memory, send <someone> } \\
\text { to the cleaners }\end{array}$ \\
\hline Covariational Conditional & $\begin{array}{l}\text { The Xer the Yer (e.g the more you think about it, } \\
\text { the less you understand) }\end{array}$ \\
\hline Ditransitive (double object) & $\begin{array}{l}\text { Subj V Óbj1 Obj2 (e.g. he gave her a fish tacos, he } \\
\text { baked her a muffin) }\end{array}$ \\
\hline Passive & $\begin{array}{l}\text { Subj aux VPpP (PP }\left(\mathrm{P}_{\mathrm{b}}\right) \text { (e.g, the armadillo mas hit } \\
\text { by a car) }\end{array}$ \\
\hline
\end{tabular}

$\left[\mathrm{VTR}_{\mathrm{i}}-\mathrm{able}_{\mathrm{Aj}} \leftrightarrow\left[\left[\mathrm{CAN} \text { BE SEM } \mathrm{i}_{\mathrm{i}} \text {-ed }\right]_{\text {PROPERTY }}\right]_{\mathrm{j}}\right.$

Morphemes are parts of constructions, but not constructions themselves.

FIGURE 22

Geert Booij has worked out a constructional account of morphology and states that morphemes are parts of constructions, but not constructions themselves. If we have, for example, a construction that has the suffix -able in English, that pattern maps onto a semantic structure, but the affix by itself does not. It can't be used by itself. Both views have advantages. If we say that morphemes are constructions, we acknowledge that they are symbolic pairings of form and meaning, and that we can maintain the idea that knowledge of language is knowledge of constructions and nothing else. If we say that morphemes are parts of constructions, we recognize that they actually need a linguistic context to be produced, a host that they can attach to, and that their meaning comes about in that specific context, but not in others. I will have more to say about morphological constructions in later lectures, so we will come back to this.

Controversy $\#_{5}$ is a clash of two views on frequency. Are associations between constructions and lexical elements measured best by raw frequencies or by a collocational measure? Anatol Stefanowitsch and Stefan Th. Gries have developed collostructional analysis as a way of finding construction-specific patterns of lexical preferences. That is, when we count the frequency of a lexical item in the context of a specific construction, we also need to take into account how often that lexical item is used elsewhere, outside of the construction. That tells us whether it is actually occurring frequently in the construction because it is attracted to that construction, or simply because it is very frequent everywhere else as well. Joan Bybee sees things differently. 


\section{Controversy \#5}

- Collostructional analysis vs. raw frequencies

- Are associations between constructions and lexical elements measured best by raw frequencies or by a collocational measure?

[A]rguing and theorizing on the basis of mere frequency data alone runs a considerable risk of producing results which might not only be completely due to the random distribution of words [in a corpus], but which may also be much less usage-based than the analysis purports to be.

Gries et al. (2005: 665)
[T] $]$ he frequency of the lexeme $L$ in the construction is the most important factor.

Bybee (2010: 97)

FIGURE 23

The position that Stefanowitsch and Gries hold can be summarized as follows (2005: 665):

[A] rguing and theorizing on the basis of mere frequency data alone runs a considerable risk of producing results which might not only be completely due to the random distribution of words [in a corpus], but which may also be much less usage-based than the analysis purports to be.

Bybee, on the other hand, defends the use of raw frequencies. She (2010: 97) states that the frequency of the lexeme $L$ in the construction, and here she means raw frequency, is the most important factor. Now, with regard to this controversy, my own position lines up with Stefanowitsch and Gries. As you will hear in the next lectures, I have been working extensively with collostructional methods. I believe that the evidence that you get from those methods actually speaks for itself.

Summing up, the five controversies that I have discussed concern the conflict between complete inheritance versus redundant representations, the importance of low-level constructions versus higher-order schemas, and the idea of constructions being forms that are paired with sound, or meanings that are paired with sound and grammatical structures. Then we have the controversy of morphemes as constructions versus morphemes as parts of constructions, and lastly, collostructional analysis versus raw frequencies. Up to this point, we have covered the basic notions of Construction Grammar, we have 
seen some strategies that allow us to define constructions, and we have learned about some controversies.

Let me finish this first lecture with an outlook on some new directions in Construction Grammar. Earlier this year, Adele Goldberg (2019) published a book with the curious title Explain Me This, which explores two theoretical notions, namely, coverage and statistical preemption. I will explain what both of these are. The central question of the book is what Goldberg calls the explain-me-this puzzle. *Explain me this is an ungrammatical sentence of English. It should be Explain this to me. Explain famously does not work in the ditransitive construction, and speakers of English know this. The question is, how do they know this? They haven't been told so by their parents. They haven't read it in a book. They somehow came to understand that it is not possible to use explain in this way. How do you learn not to say things? That is a veritable scientific puzzle that Goldberg tries to solve. She asks, how is it possible that speakers of a language accept certain utterances that they have never heard before as completely idiomatic, while they reject other utterances as simply impossible and ungrammatical? Consider these two examples. Vernon tweeted to say she does not like us is a sentence that you may have never seen before, but that speakers judge to be possible. *She considered to say something is also something that you may never have heard before, but here speakers will insist that this does not sound right. How do these different judgments come about? The phenomenon, I should add, is not limited to English. If you do not have intuitions on these two sentences, do not worry. In any language, there are new ways of saying things that are easily possible. There are some unusual word combinations that are fine, and then there are other combinations that speakers will reject as not possible. Goldberg tries to explain why this is so.

She argues that there are two central factors. The first is coverage, which involves the mutual similarity between different instantiations of a construction. The second is statistical preemption, which relates to the idea of competition between constructions. Let me say a bit more about coverage first. Coverage can be broken down into the following ideas. For any given construction, there is a degree of mutual similarity between different instantiations of a construction. Speakers have highly detailed linguistic memories. That is the basic idea of usage-based linguistics that I presented to you. For any construction that we use, we keep a record of the examples that we hear, how similar they are to each other and where any new example that we find fits in. That applies across all levels of linguistic structures. That applies to you adjusting to my vowels. It applies to speakers hearing a new and original instance of the ditransitive construction. When we hear a new token of language use, we integrate that into our old memories. If the new token is just like everything 
we've ever heard before, our overall representations are not changed. But when this new instance is different, then it subtly shifts our representations to a new space. Our linguistic categories and their representations are continually formed and updated, so that our knowledge of language continually changes over time. That means that any construction that we are talking about has a certain quality of coverage. Let me make this more concrete.

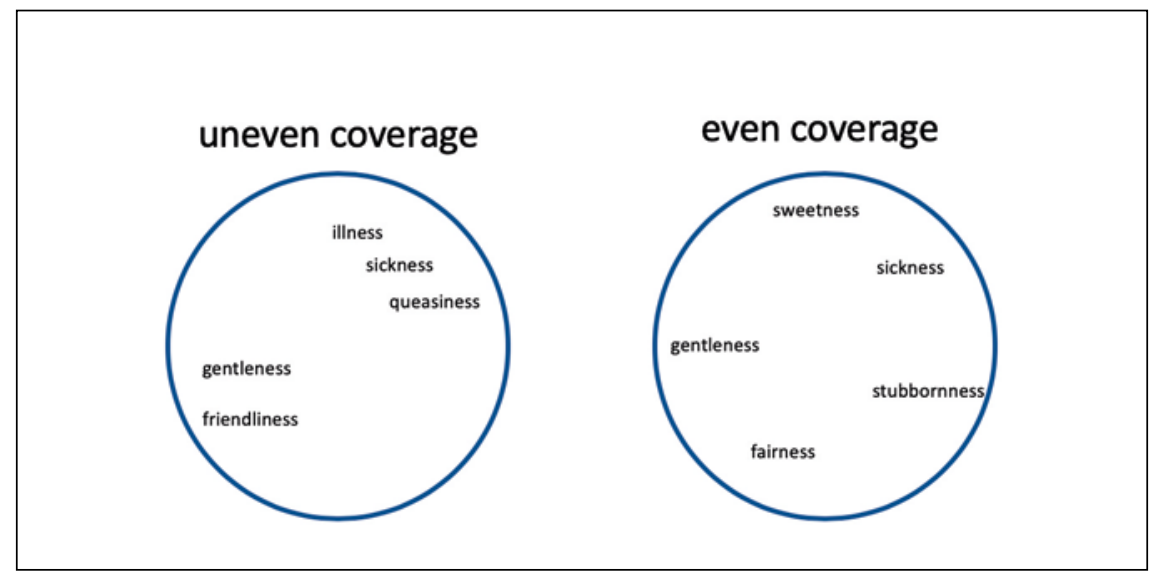

FIGURE 24

Here we have two hypothetical scenarios for which I am using the English derivational suffix -ness as an example. You can think of this as two speakers and their respective experience with the English -ness construction. In the first case, the construction has exactly five different types, and these five fall into two clearly defined groups. We have gentleness and friendliness, which are both about someone being an agreeable person. Then the other three, illness, sickness, and queasiness, all relate to not feeling well in different ways. This is what Goldberg would call "uneven coverage". The distribution of types is not very homogeneous, instead there are clusters.

In the second case, we have a speaker who has a very different experience with the -ness construction. We have the same construction with the same number of types, five different words, but those types all convey very different ideas. We have gentleness, sweetness, sickness, stubbornness and fairness, which encode very different meanings. This would illustrate what Goldberg calls 'even coverage'. All words are approximately the same in terms of their similarity and distance from each other.

Now imagine what happens if these two speakers that we have here encounter a new type of the construction, for example, the word carelessness. In the first case, carelessness is situated right between these two clusters that the 


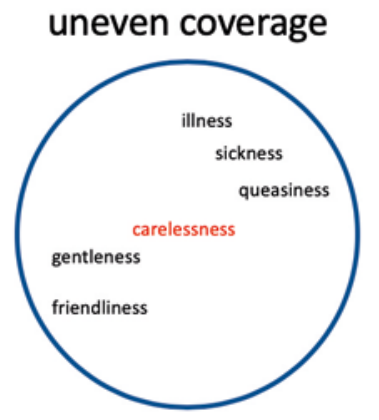

even coverage

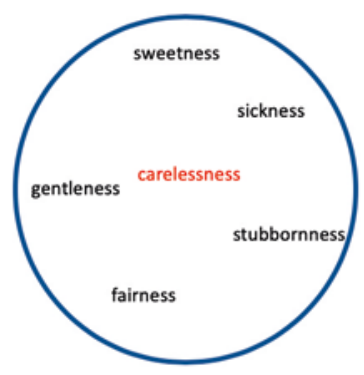

FIGURE 25

speaker has come to represent. Carelessness requires the speaker to update and change their knowledge of the construction in substantial ways. They realize that there are instances of the construction between the established clusters. Instead of the two well-defined clusters, there is now a more continuous semantic spectrum.

In the second case, the distance of every type to every other type is still more or less the same. Nothing much changes, just one more type has been added to a semantic spectrum that is already semantically diverse. The implication is that constructions with even coverage allow new types much more easily than constructions with uneven coverage.

Going back to the earlier state of affairs, which speaker do you think will be more inclined to form new types, to come up with new words that have this -ness suffix? The one on the left will probably produce types that relate to these two clusters, but not new types that would be situated between them, in the middle of the semantic space. That is what is meant by the term coverage.

The second notion is captured by the term statistical preemption. Statistical preemption relates to the idea of higher-order schemas that I talked about earlier in connection with the work of Florent Perek. The idea is that speakers form generalizations over sets of functionally similar constructions, like the ditransitive (John give Mary the book) and the prepositional dative (John gave the book to Mary). Speakers realize that these two constructions are similar, and they keep track of the frequencies of lexical elements that occur in them. They take note of asymmetries that they view as striking, as conspicuous. This can actually explain how speakers learn not to say certain things. To make this idea more concrete, let me show you an example of how Boyd and Goldberg (2011) investigated this. 
On the following slides, you will see moving pictures, and I just want you to say silently in your mind what happens.

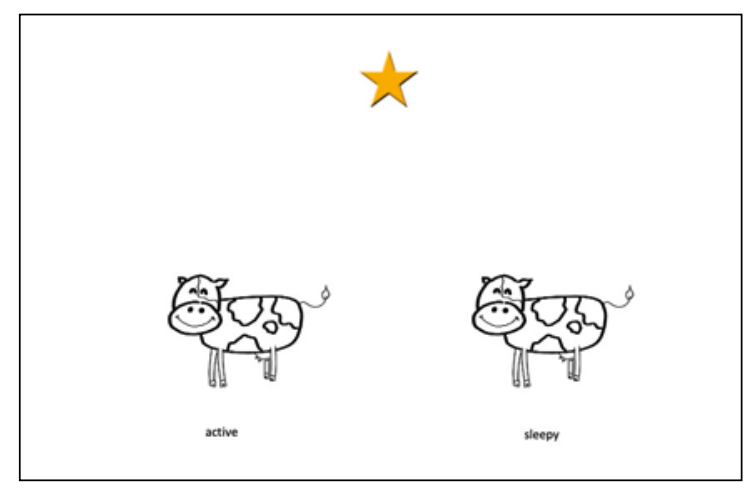

FIGURE 26

On this slide you see two cows. There is an active cow and a sleepy cow. Participants in this experiment would see something like this [The left cow moves to the star], and they would have to describe the depicted event by saying The active cow moves to the star.

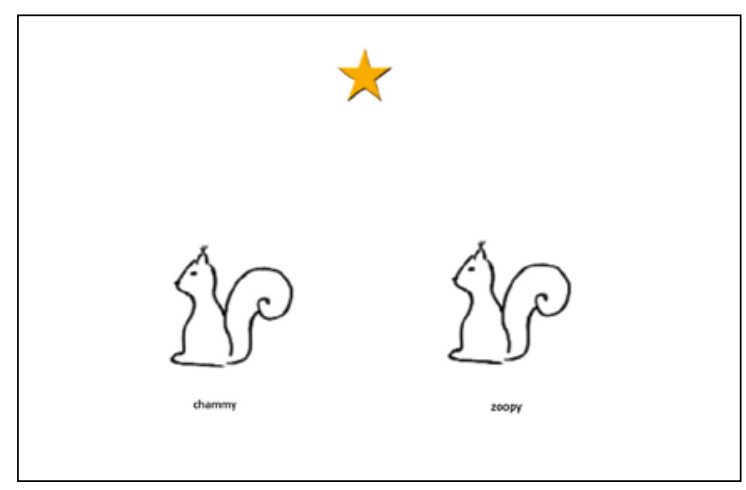

FIGURE 27

This slide shows two squirrels. These words below them are descriptions, not names. They are novel adjectives that are meant to describe these squirrels. You show participants this kind of scenario [The right squirrel moves to the star], and they would say The zoopy squirrel moves to the star. 


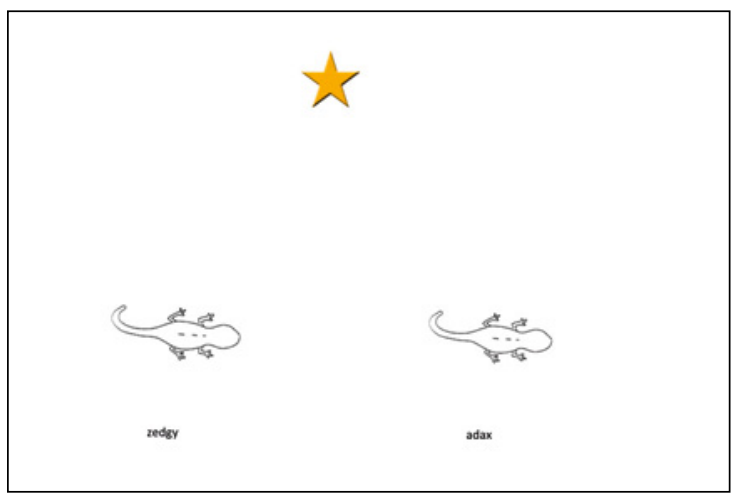

FIGURE 28

Here is another example of the experimental stimuli. The slide shows two lizards, and one of them moves to the star [The right lizard moves to the star]. Here speakers might say The adax lizard moves to the star or The lizard that is adax moves to the star.

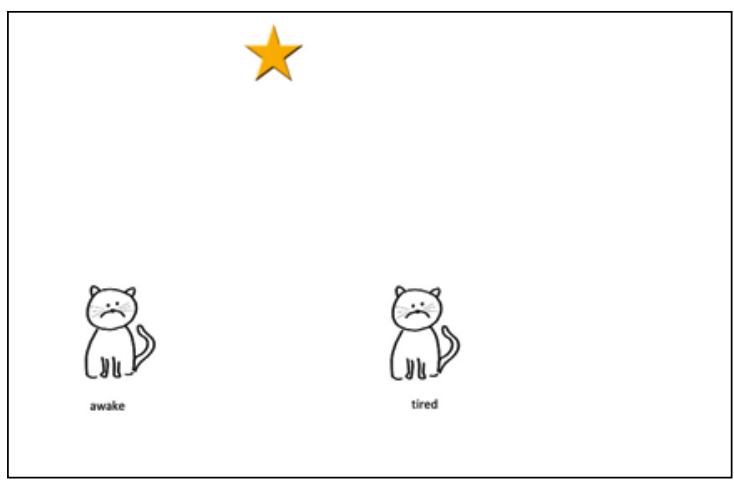

FIGURE 29

Consider this final example. This slide shows two kittens. When the left of them moves to the star, speakers would say The kitten that is awake moves to the star.

The adjectives that Boyd and Goldberg (2011) used in this experiment have certain characteristics. The stimulus with the kittens features one of the adjectives that I discussed earlier, so-called $a$-adjectives like asleep or awake or alive. They can only be used predicatively in English. It is only possible to say The kitten that is awake moves to the star, but you cannot say *The awake kitten moves to the star.

The most interesting type of stimulus in Boyd and Goldberg's (2011) experiment is illustrated by the one with the two lizards. Do the participants say The adax lizard moves to the star, or do they recognise adax as one of these 
a-adjectives that have to be treated in a special way? Do they prefer to say The lizard that is adax move to the star? If they do, that means that they have formed a generalization on the basis of other $a$-adjectives that they have heard, and they project the constraints of that $a$-adjective construction to new adjectives that they haven't heard so far. As a consequence, they would avoid *the adax lizard, because they assume it to be ungrammatical. Think about that. They have intuitions about ungrammatical uses of a word that they have never heard before. They have learned not to say certain things, despite the fact that they do not have any active evidence apart from the distributional knowledge of what they've heard so far.

\section{stimuli}

\begin{tabular}{llll}
\multicolumn{1}{c}{ FAMILIAR } & \multicolumn{1}{c}{ NOVEL } \\
\multicolumn{1}{c}{ N- } & \multicolumn{1}{c}{ NON- $a-$} & \multicolumn{1}{c}{ NON- $a$ - } \\
afloat (sinking) & floating (sinking) & ablim (zecky) & chammy (zoopy) \\
afraid (brave) & frightened (brave) & adax (zedgy) & flitzy (zappy) \\
alive (dead) & living (dead) & afraz (zibby) & gecky (zunderful) \\
asleep (vigilant) & sleepy (vigilant) & agask (zintesting) & slooky (zinky)
\end{tabular}

TABLE 1. Critical target adjective labels; foil labels are in parentheses.

FIGURE 30

Here are the different types of stimuli that Goldberg and Boyd (2011: 66) used. The experiment cleverly cross-cuts ordinary non- $a$ adjectives like sleepy with made-up artificial adjectives such as chammy or zoopy. The question is whether speakers treat a made-up adjective like adax like ordinary adjectives such as sleepy. Do they say The lizard that is adax moves to the star, or do they say The adax lizard moves to the star? They haven't heard the word adax before, so they might assume that it works like any other adjective, but the crucial conclusion is that they do not.

The main result of the study can be seen in the graph on the slide here (Boyd and Goldberg 2011: 69). The important piece of information is the height of the bars in this chart. The higher the bar, the more speakers in the experiments actually chose a description that involved the attributive adjective like the active cow. If the bars are low, that means that the speaker rather chose a relative clause construction like the cow that is active. 


\section{results}

- main effect of adjective type

- a-adjectives typically used with relative clauses

- main effect of familiarity

- novel adjectives used more often attributively

- interaction of adjective type and familiarity

- unfamiliar a-adjectives less often used with relative clauses than familiar a-adjectives

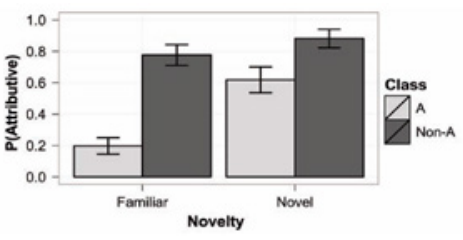

FIGURE 31

What you can see is that there is a major difference between non- $a$ adjectives in dark gray, which have a large proportion of attributive uses like the active $c o w$, and $a$-adjectives in light gray like awake or asleep. In the familiar condition, very few of these are used before the noun. There are some people who say the awake kitten, and but not many. The crucial category is shown by the second light gray bar. These are the adjectives like adax. You see that speakers are influenced by the presence of an initial $a$. They say the adax lizard less often than could be hypothesized. To them *the adax lizard does not sound quite right, and this shows that they have generalized from awake and asleep to a new word, namely, adax. They have learned not to say a certain thing.

In the context of statistical preemption, there is one further controversy that I would like to mention, which concerns the way in which statistical preemption is supposed to work. I would like to discuss two competing accounts, one by Adele Goldberg herself and one proposed by Anatol Stefanowitsch. Stefanowitsch uses the term 'negative entrenchment' for his account. What is the difference between those two? Goldberg's (2019) position is that statistical preemption works in such a way that speakers reject the creative use of a construction when there is an alternative that they know about. When speakers know a conventionalized and alternative expression, they won't get creative. Speakers know that the child that is afraid works fine. They have never heard the phrase *the afraid child, which would be a conceivable alternative, and as a consequence they shy away from using $a$-adjectives before the noun. Likewise, the speakers have experienced the verb want with a to-infinitive complementation 
pattern lots of times, as in She wanted to say something. By contrast, want with an -ing type complement, as in *She wanted saying something, is never encountered, despite the fact that it would be a possible alternative. The asymmetry between these alternatives is what leads speakers to disprefer * She wanted saying something. For Goldberg, it is crucial that there is competition between two alternatives constructions that mean approximately the same thing.

Stefanowitsch (2011) argues a point that is subtly different. For him, learning not to say a certain thing is not necessarily due to competing alternatives. Rather, negative entrenchment for Stefanowitsch works in such a way that speakers reject the creative use of a construction when they have heard that construction frequently in other contexts, but never before in the creative one. Say famously does not work in the ditransitive construction. According to Stefanowitsch, that is because say is a very frequent verb. It occurs in many constructions, but the speaker has never heard it in the ditransitive.

With regard to this controversy, I am actually happy to let you know that I am currently involved in experimental work together with Adele Goldberg where we try to test the merits of both points of view. I do not think they are mutually exclusive. I am convinced that statistical preemption works in a way that Goldberg proposes, but it remains to be seen if negative entrenchment also works.

The last idea I'd like to present this morning is called constructional contamination. What is it? It is

an effect whereby a subset of instances of a target construction is affected in its realization by a contaminating construction, because of a coincidental resemblance between the superficial strings of instances of the target construction and a number of instances in the contaminating construction.

PIJPOPS AND VAN DE VELDE 2016: 543

Let me unpack that definition and explain how constructional contamination works in practice. Constructional contamination can affect a target construction such as the English passive. Here's an example sentence of the passive, the disease was sexually transmitted. We have a participle and an adverb in this particular sequence. The adverb comes first, the participle follows. This target construction may be influenced by a contaminating construction that is superficially similar. In the case of the passive, a potentially contaminating construction is a noun phrase construction, where we see the exact same order of adverb and participle, first sexually, then transmitted. The sequence 
hypothesis: high frequencies in the NP >> preference for ADV-PPART

\begin{tabular}{|c|c|c|c|c|}
\hline adverb & participle & passive (ADV-PPART) & passive (PPART-ADV) & complex modifier NP \\
\hline well & known & 1594 & 4 & 110 \\
\hline best & known & 957 & 7 & 212 \\
\hline also & found & 608 & 12 & 169 \\
\hline widely & used & 501 & 55 & 11 \\
\hline randomly & assigned & 444 & 33 & 39 \\
\hline often & called & 407 & 1 & 93 \\
\hline also & included & 283 & 2 & 205 \\
\hline 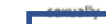 & Almedrad & 201 & & הב \\
\hline privately & owned & 151 & 6 & 290 \\
\hline puentery & traver & 00 & 2 & 300 \\
\hline well & established & 376 & 3 & 31 \\
\hline highly & regarded & 122 & 2 & 265 \\
\hline dimly & lit & 64 & 1 & 297 \\
\hline randomly & selected & 308 & 38 & 13 \\
\hline clearly & defined & 105 & 6 & 241 \\
\hline democratically & elected & 42 & 12 & 284 \\
\hline hard & hit & 91 & 245 & 1 \\
\hline specifically & designed & 187 & 136 & 8 \\
\hline better & prepared & 331 & 1 & 1 \\
\hline often & seen & 310 & 3 & 2 \\
\hline
\end{tabular}

Table 2: Twenty frequent adverb-participle collocations

FIGURE 32

of adverb and participle appears in two different syntactic contexts. One may influence the other. What is crucial is that the target construction, the passive, allows variation. Speakers of English can say The disease was sexually transmitted or The disease was transmitted sexually. Both are grammatically possible, but in the contaminating construction, there is no variation, only the adverb initial order is possible.

A relevant question to ask is whether frequent usage of sexually transmitted in the noun phrase construction can lead to a relative preference of that order, adverb first and then participle, in the passive. This can be tested empirically.

In work that I have done together with Susanne Flach, we have examined corpus data to check whether high frequencies of an adverb participle combination in the noun phrase construction correlate with the preference for adverb initial order in the passive. Our results indicate that combinations that occur frequently in the noun phrase will have a contaminating effect on the passive. For example, the combination privately owned is a combination that is very frequent in the noun phrase construction, and when we compare the frequencies in the passive, we see that there is a strong asymmetry. Privately owned is much more frequent in the passive than owned privately. Speakers frequently say The company is privately owned, but the alternative The company is owned privately is a lot less frequent. You can explain that in terms of this combination of the noun phrase construction, which contaminates the use of the passive construction.

To conclude, data from the English passive offers support for the idea of constructional contamination, which means that frequent collocations in one 
construction can influence syntactic variation in another syntactically homonymous construction. This means that in the speaker's knowledge, syntactic schemas are connected, and superficial structural similarities are enough for speakers to form and entertain connections between constructions.

I am coming to an end. This morning, I have given you ten basic ideas of construction grammar, which I think sum up the enterprise. I have given you a number of strategies that allow you to identify constructions. I have talked about five controversies where architects of construction grammar are currently debating how we should think of certain notions. Finally, I have outlined a couple of new developments. Researchers in Construction Grammar are detecting the limits of constructional productivity with the notions of coverage and statistical preemption, and they are detecting patterns of association through evidence of constructional contamination. There is one new development that will form the backbone of every lecture that follows from now on, namely, the application of constructional approaches to the study of language change, which is what we do in Diachronic Construction Grammar.

Starting with Lecture 2, I will thus focus on language change. I look forward to seeing you this afternoon. Thank you very much for your attention. 